\title{
Impact of Changing Activation Sequence on Bipolar Electrogram Amplitude for Voltage Mapping of Left Ventricular Infarcts Causing Ventricular Tachycardia
}

\begin{abstract}
Brunckhorst, Corinna B ; Delacretaz, Etienne ; Soejima, Kyoko ; Maisel, William H ; Friedman, Peter L
\end{abstract} ; Stevenson, William G

\begin{abstract}
Introduction: Wavefront direction is a determinant of bipolar electrogram amplitude that could influence identification of low amplitude regions indicating infarction or scar. Methods: To assess the importance of activation sequence on electrogram amplitude 11 patients with prior infarction and ventricular tachycardia were studied. At 819 left ventricular sites bipolar electrograms were recorded during atrial pacing and ventricular pacing, followed by unipolar pacing with a stimulus of $10 \mathrm{~mA}$ at 2 ms. Sites with a pacing threshold $>10 \mathrm{~mA}$ were designated electrically unexcitable scar. Results: Areas of low voltage $(1.5 \mathrm{mV})$ were present in all patients. Atrial paced and ventricular paced electrogram amplitudes were strongly correlated $(\mathrm{r}=0.77 ; \mathrm{P}<0.0001)$. Changing the activation sequence (from atrial pacing to ventricular pacing) produced a $>50 \%$ change in electrogram amplitude at $28 \%$ of sites and $\mathrm{a}>100 \%$ change at $10 \%$ of sites, but only $8 \%$ of sites had an electrogram amplitude classified as abnormal $(1.5 \mathrm{mV})$ with one activation sequence and normal $(>1.5 \mathrm{mV})$ with the other activation sequence. Electrically unexcitable scar (6\% of sites) was associated with lower electrogram amplitude but could not be reliably identified based on electrogram amplitude alone for either activation sequence. Conclusion: Voltage maps created with bipolar recordings using these methods should be relatively robust depictions of abnormal ventricular regions despite variable catheter orientation and activation sequences that might be produced by different rhythms
\end{abstract}

DOI: https://doi.org/10.1007/s10840-005-6549-z

Posted at the Zurich Open Repository and Archive, University of Zurich

ZORA URL: https://doi.org/10.5167/uzh-156303

Journal Article

Published Version

Originally published at:

Brunckhorst, Corinna B; Delacretaz, Etienne; Soejima, Kyoko; Maisel, William H; Friedman, Peter L; Stevenson, William G (2005). Impact of Changing Activation Sequence on Bipolar Electrogram Amplitude for Voltage Mapping of Left Ventricular Infarcts Causing Ventricular Tachycardia. Journal of Interventional Cardiac Electrophysiology, 12(2):137-141.

DOI: https://doi.org/10.1007/s10840-005-6549-z 


\title{
Impact of Changing Activation Sequence on Bipolar Electrogram Amplitude for Voltage Mapping of Left Ventricular Infarcts Causing Ventricular Tachycardia
}

\author{
Corinna B. Brunckhorst, ${ }^{1}$ Etienne Delacretaz, ${ }^{2}$ \\ Kyoko Soejima, ${ }^{3}$ William H. Maisel, ${ }^{3}$ Peter L. \\ Friedman, ${ }^{3}$ and William G. Stevenson ${ }^{3}$ \\ ${ }^{1}$ Cardiovascular Center, University Hospital Zurich, \\ Switzerland; ${ }^{2}$ Cardiovascular Center, University Hospital Berne, \\ Switzerland; ${ }^{3}$ Cardiovascular Division, Brigham and Women's \\ Hospital, Boston, USA
}

\begin{abstract}
Introduction: Wavefront direction is a determinant of bipolar electrogram amplitude that could influence identification of low amplitude regions indicating infarction or scar.

Methods: To assess the importance of activation sequence on electrogram amplitude 11 patients with prior infarction and ventricular tachycardia were studied. At 819 left ventricular sites bipolar electrograms were recorded during atrial pacing and ventricular pacing, followed by unipolar pacing with a stimulus of $10 \mathrm{~mA}$ at $2 \mathrm{~ms}$. Sites with a pacing threshold $>10 \mathrm{~mA}$ were designated electrically unexcitable scar.

Results: Areas of low voltage $(\leq 1.5 \mathrm{mV})$ were present in all patients. Atrial paced and ventricular paced electrogram amplitudes were strongly correlated $(r=0.77 ; p<0.0001)$. Changing the activation sequence (from atrial pacing to ventricular pacing) produced a $>\mathbf{5 0} \%$ change in electrogram amplitude at $28 \%$ of sites and a $>100 \%$ change at $10 \%$ of sites, but only $8 \%$ of sites had an electrogram amplitude classified as abnormal $(\leq 1.5 \mathrm{mV})$ with one activation sequence and normal $(>1.5 \mathrm{mV}$ ) with the other activation sequence. Electrically unexcitable scar (6\% of sites) was associated with lower electrogram amplitude but could not be reliably identified based on electrogram amplitude alone for either activation sequence.

Conclusion: Voltage maps created with bipolar recordings using these methods should be relatively robust depictions of abnormal ventricular regions despite variable catheter orientation and activation sequences that might be produced by different rhythms.
\end{abstract}

Key Words. ventricular tachycardia, myocardial infarction, reentry, catheter ablation, electrogram voltage, direction of wavefront propagation

\section{Introduction}

Ventricular tachycardia (VT) late after myocardial infarction is usually due to reentry involving regions of infarction. The amplitude of electrograms recorded from infarct regions is reduced
$[1,2]$. Three-dimensional "voltage maps," that plot the location of these areas have emerged as a useful tool for guiding ablation of scar related VT. These maps have been created with bipolar recordings and a normal amplitude of $>1.5 \mathrm{mV}$ is commonly employed, based on the data of Marchlinski and coworkers showing that approximately $95 \%$ of sites in normal ventricles exceed this amplitude [2]. With bipolar recordings, the orientation of the recording bipole in relation to the propagating wavefront influences electrogram amplitude. This phenomenon has the potential to produce variable electrogram amplitude depending on the direction of wavefront propagation at the recording site and has not been previously studied with catheter mapping in humans. The issue is clinically relevant because many patients require mapping during ventricular pacing rather than sinus rhythm and some alternate between paced, sinus rhythm, and ventricular tachycardia during mapping procedures.

The purpose of this study is to investigate the impact of a change in activation sequence (from atrial pacing to right ventricular pacing) on left ventricular electrogram amplitude during creation of voltage maps.

\section{Methods}

\section{Patients}

Left ventricular mapping was performed in 11 male patients (mean age $68 \pm 9$ years) who had at

Address for correspondence: Dr. Corinna B. Brunckhorst, MD, Instructor in Cardiology, Cardiovascular Center, University Hospital Zurich, Ramistr. 100, Zurich 8091, Switzerland. E-mail: corinna.brunckhorst@usz.ch

Received 28 June 2004; accepted 26 October 2004 
least one remote ( $>2$ months) myocardial infarction with a mean left ventricular ejection fraction of $25 \pm 9 \%$ and a range of 15 to $40 \%$, who were referred for catheter ablation of recurrent sustained monomorphic VT ( $\geq 2$ episodes in the preceding 6 months).

\section{Mapping}

Catheter mapping and ablation and data collection were performed prospectively according to protocols approved by the institutional review board of Brigham and Women's Hospital, Boston, MA after obtaining written informed consent. Left ventricular (LV) access was achieved with a retrograde aortic or transseptal approach. Systemic anticoagulation was maintained with heparin.

Mapping used the non-fluoroscopic electroanatomic mapping system CARTO ${ }^{\circledR}$ (Biosense Webster, Diamond Bar, CA) [3]. Electrograms recorded from the catheter are stored in combination with their exact anatomic location with a spatial accuracy of $\pm 0.8 \mathrm{~mm}$ [4]. The $7 \mathrm{~F}$ deflectable, quadripolar catheter has a $4 \mathrm{~mm}$ ablation tip and a spacing of 1,7 and $4 \mathrm{~mm}$ between the distal, middle and proximal electrodes, respectively. Bipolar electrograms were recorded from the distal electrode pair and filtered at $10-400 \mathrm{~Hz}$. At each stable ventricular site electrograms were recorded during atrial pacing, then during right ventricular pacing. Pacing was then performed from the mapping catheter (pace mapping) at one consistent paced cycle length for each patient, between 500-700 ms. Pace mapping utilized unipolar stimuli with a strength of $10 \mathrm{~mA}$ and pulse width of $2 \mathrm{~ms}$ [5]. This fixed stimulus strength was chosen due to time constraints of ventricular mapping and because we have extensive experience pacing at this stimulus strength for mapping purposes and have observed reliable capture in normal and abnormal hearts. Catheter stability was assessed from fluoroscopy, the CARTO mapping system, electrogram stability, and consistent capture or lack of capture with pacing. If pacing did not capture the position of the catheter was reviewed with biplane fluoroscopy and assessed for possible poor contact. Sites where pacing capture was intermittent or where motion of the catheter or gentle additional pressure moved the catheter suggesting that it was not in physical contact with the endocardium were excluded from analysis.

Custom software allowed the intracardiac electrograms and 12 surface ECG leads for each site to be digitized and stored during atrial pacing, right ventricular pacing, and pace mapping before moving the catheter to the next mapping site. The maximum electrogram amplitude (peak to peak) from the distal electrode pair of the mapping catheter was automatically measured and displayed on corresponding maps. Sites were manually reviewed to ensure exclusion of pacing stimuli from amplitude measurements.

\section{Statistical Analysis}

All values are expressed as mean \pm standard deviation. Continuous variables are compared using the two-tail unpaired t-test. Correlation coefficients were calculated with the Pearson test. Discrete variables were compared using the Fisher exact test. Generalized estimating equations were used to adjust for multiple observations in individual patients. [6]. A two-sided probability value of $<0.05$ was considered significant. Calculations were performed using SAS Statistical Software (Version 6.12, Cary NC).

\section{Results}

All patients had large regions of low $(\leq 1.5 \mathrm{mV})$ voltage electrograms consistent with infarcts. Infarct locations were inferior or posterior in 8, anterior in 1 , and in two discrete regions in two patients (posterobasal and inferoseptal in one, and anteroapical and inferoseptal in the other).

A total of 819 sites (mean per patient $75 \pm$ 24) met criteria for analysis, of which $59 \%$ had an amplitude $\leq 1.5 \mathrm{mV}$ during atrial pacing and $59 \%$ during right ventricular pacing. Changing the ventricular activation sequence by pacing from the atrium as compared to the right ventricle had relatively little effect on maximal peak-topeak electrogram amplitudes, particularly at sites with very low amplitude electrograms (Table 1 and Figs. 1-3). There was a strong correlation of electrogram amplitude between atrial pacing and right ventricular pacing $(r=0.77 ; p<0.0001$; Fig. 3). Considering $>1.5 \mathrm{mV}$ as normal, $8 \%$ of sites had an abnormal electrogram amplitude $(\leq 1.5 \mathrm{mV})$ with one activation sequence and normal ( $>1.5 \mathrm{mV}$ ) with the other activation sequence. Although substantial changes in amplitude did occur, these were more frequent in normal than abnormal regions. Changing the activation sequence changed electrogram amplitude by $>50 \%$ at a similar number of low amplitude and normal amplitude sites (Table 1), with a change $>1.5 \mathrm{mV}$ much more often at the normal amplitude sites.

With pacing from the ablation catheter capture was present (pacing threshold $\leq 10 \mathrm{~mA}$ ) at 771 (94\%) sites. The $48(6 \%)$ sites where pacing did not capture were classified as electrically unexcitable scar [5]. Electrically unexcitable sites had lower electrogram amplitude than excitable sites during atrial pacing $(0.9 \pm 0.8$ vs. $2.1 \pm 2.5 \mathrm{mV} ; p=0.004)$ and during right ventricular pacing $(0.8 \pm 1.0 \mathrm{vs}$. $2.2 \pm 2.6 \mathrm{mV} ; p=0.002$ ). A clearly definable amplitude threshold for identifying electrically 
Table 1. Impact of a change in wavefront direction on electrogram voltage amplitude

\begin{tabular}{|c|c|c|c|}
\hline & & \multicolumn{2}{|c|}{ Right ventricular pacing } \\
\hline & $\begin{array}{l}\text { Electrogram voltage } \\
\text { amplitude }\end{array}$ & $\begin{array}{l}>1.5 \mathrm{mV} \\
n=335\end{array}$ & $\begin{array}{l}\leq 1.5 \mathrm{mV} \\
n=484\end{array}$ \\
\hline \multirow[t]{3}{*}{ Atrial pacing } & $\begin{array}{l}>1.5 \mathrm{mV}, n=330 \\
\leq 1.5 \mathrm{mV}, n=489\end{array}$ & $\begin{array}{r}33 \% \\
8 \%\end{array}$ & $\begin{array}{r}8 \% \\
51 \%\end{array}$ \\
\hline & & \multicolumn{2}{|c|}{ Right ventricular pacing } \\
\hline & & $\Delta>50 \%$ & $\Delta>1.5 \mathrm{mV}$ \\
\hline \multirow[t]{4}{*}{ Atrial pacing } & $>1.5 \mathrm{mV}, n=330$ & $29 \%$ & $34 \%$ \\
\hline & $\leq 1.5 \mathrm{mV}, n=489$ & $28 \%$ & $4 \%$ \\
\hline & & \multicolumn{2}{|c|}{ Atrial pacing } \\
\hline & & $\Delta>50 \%$ & $\Delta>1.5 \mathrm{mV}$ \\
\hline \multirow[t]{2}{*}{ Right ventricular pacing } & $>1.5 \mathrm{mV}, n=335$ & $30 \%$ & $36 \%$ \\
\hline & $\leq 1.5 \mathrm{mV}, n=484$ & $29 \%$ & $3 \%$ \\
\hline
\end{tabular}

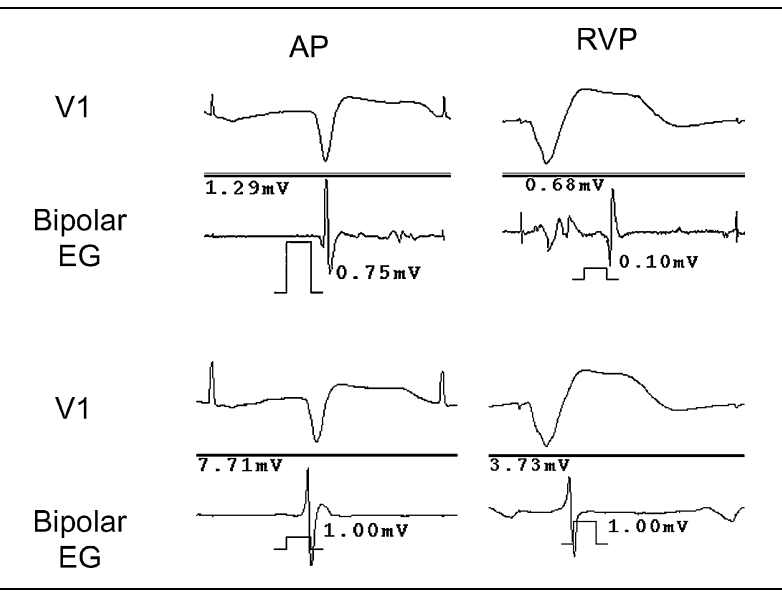

Fig. 1. Recordings from a mapping site in an infarct area (top) and a normal region (bottom) are shown. At both sites the left panel shows recording during atrial pacing (AP) and the right panel shows the recording during ventricular pacing (VP). Each panel shows a single surface ECG lead (V1) and intracardiac electrogram with a calibration signal and the measured peak to peak electrogram amplitude in $\mathrm{mV}$ on the left. The calibration is adjusted based on an automatic algorithm. In the abnormal area (top panels) maximal peak to peak electrogram amplitude is 1.29 and $0.68 \mathrm{mV}$ during atrial and ventricular pacing respectively, remaining $\leq 1.5 \mathrm{mV}$ in both cases. In the normal area (bottom panels) maximal peak to peak electrogram amplitude changes substantially from 7.71 to $3.73 \mathrm{mV}$ when changing the activation sequence from atrial to ventricular pacing, but remains $>1.5 \mathrm{mV}$ in both cases $(E G=$ electrogram $)$.

unexcitable scar was not present for either atrial or ventricular paced electrograms. Pacing captured at $96 \%$ of sites where the electrogram amplitude in atrial pacing was $>1 \mathrm{mV}$, at $91 \%$ of sites where the electrogram amplitude was $\leq 1 \mathrm{mV}$, at $89 \%$ sites where electrogram amplitude was $\leq 0.5 \mathrm{mV}$ and at $86 \%$ sites where electrogram amplitude was $\leq 0.1 \mathrm{mV}$. Results were similar for electrogram amplitudes recorded during right ventricular pacing; pacing captured at $97 \%$ of sites $>1$ $\mathrm{mV}$, at $90 \%$ of sites $\leq 1 \mathrm{mV}$, at $88 \%$ of sites $\leq 0.5$ $\mathrm{mV}$ and at $81 \%$ of sites $\leq 0.1 \mathrm{mV}$.

\section{Discussion}

Reentry circuits causing VT after myocardial infarction form in the border of infarcts where surviving myocyte bands connect the infarct region and the surrounding tissue [7]. These regions contain areas of abnormal slow conduction as well as fibrous scar creating regions of conduction block [8]. Voltage maps are useful for identifying infarct regions and guiding catheter ablation. $[1,2,5,9]$. Marchlinski and coworkers, using the same recording methods employed in our study in 4 patients without structural heart disease found that $95 \%$ of all LV electrograms had an amplitude $>1.5 \mathrm{mV}$ [2]. They used this cutoff to identifying infarct regions in 9 patients with prior myocardial infarction and VT.

Voltage maps can be created from unipolar recordings, but unfiltered unipolar recordings are heavily weighted by "far-field" signals from adjacent myocardium, such that electrogram amplitude may reflect the surrounding mass of more normal myocardium rather than the presence or absence of surviving myocytes beneath the recording electrode. Bipolar recordings subtract out much of this far-field signal and were employed in the present and previous studies relating electrogram amplitude to the infarct region [1,2,9-12].

The ability of wavefront direction to influence electrogram amplitude is a potential limitation 


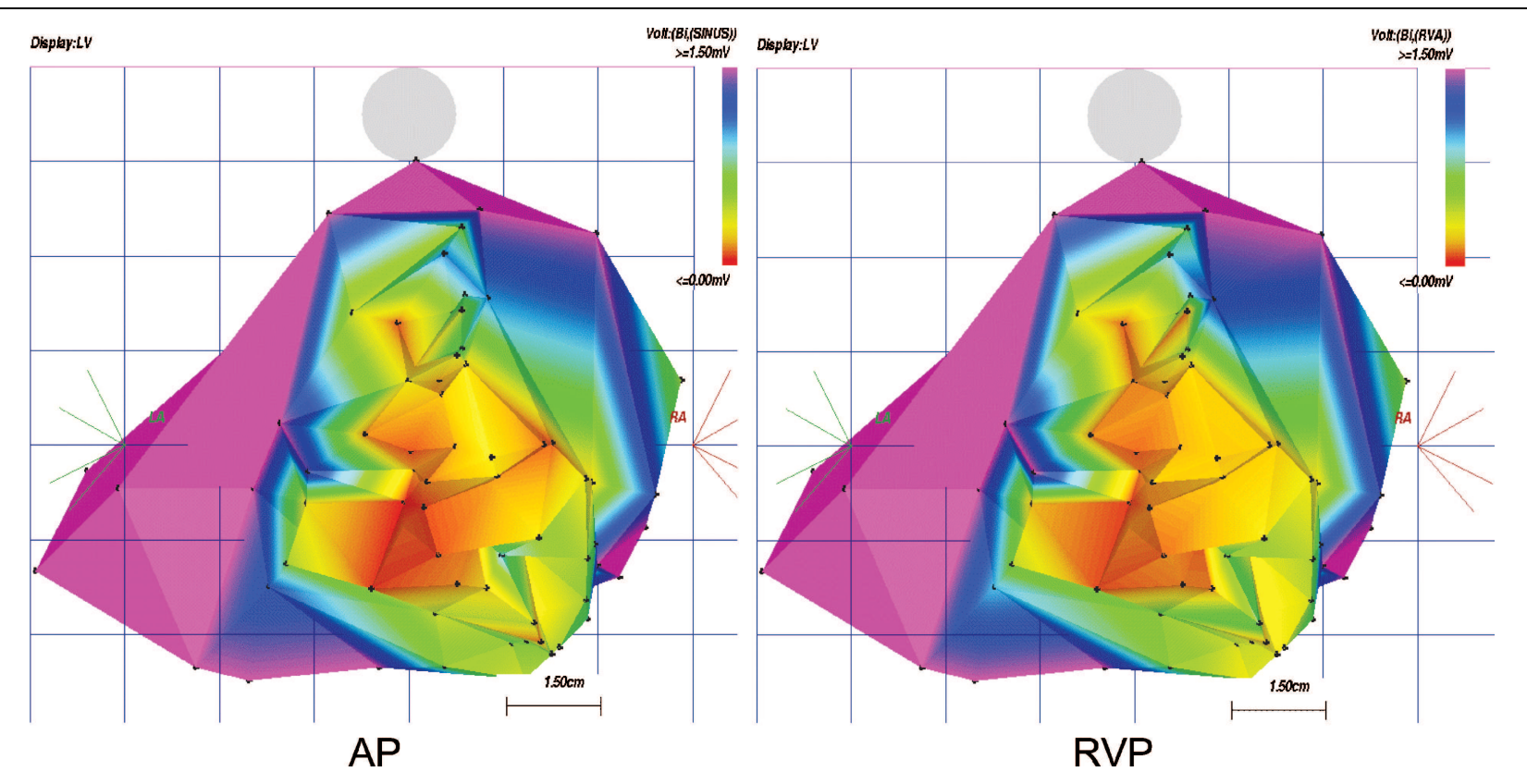

Fig. 2. Electroanatomic bipolar voltage maps of the left ventricle in atrial pacing and right ventricular pacing are shown in a posterior projection. Electrogram amplitude $(\mathrm{mV})$ is color coded from red $(0 \mathrm{mV})$ to yellow, green, and blue to purple $(\geq 1.5 \mathrm{mV})$. A large posterior low voltage region is present and similar during both pacing modes.

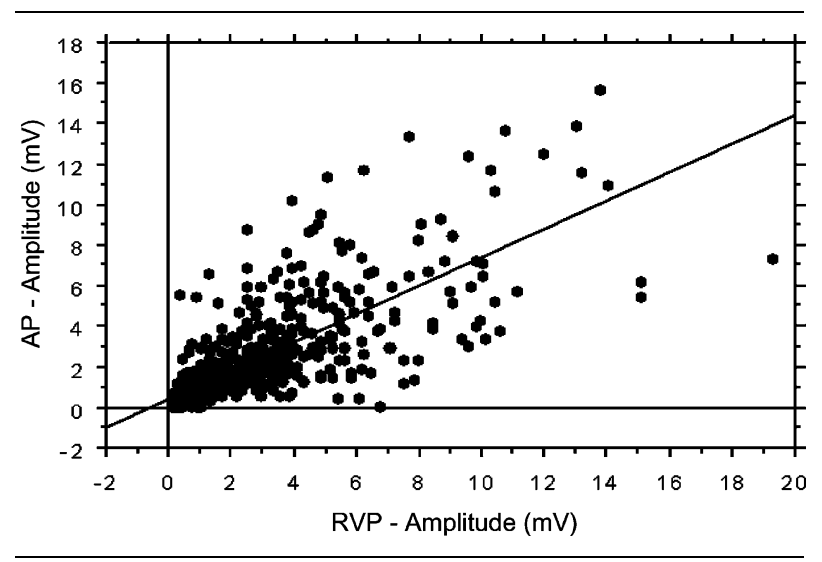

Fig. 3. Shown is a plot of the electrogram amplitude during right ventricular pacing $(R V P-V)$ ( $x$-axis) versus atrial pacing $(A P-V)\left(y\right.$-axis). $\left(r=0.77 ; y=0.47+0.70^{*} x\right)$.

of bipolar recordings. A wavefront that propagates perpendicular to the axis of the recording dipole will have lower amplitude than a wavfront, which propagates longitudinally to the recording dipole [13]. We studied the impact of a change in wavefront direction by comparing electrograms during atrial pacing with those obtained from the identical site during right ventricular pacing. Variation of electrogram amplitude $>50 \%$ did occur at $28 \%$ of all sites, but the absolute amplitude in low voltage regions did not change markedly, and there was little effect on the size of the low voltage region (Fig. 2) when a cutoff of $1.5 \mathrm{mV}$ was used. Thus, our findings suggest that voltage maps using this cutoff should be relatively robust, and little influenced by direction of wavefront propagation produced by a different rhythm or pacing site, although it is still possible that changes in activation could induce small changes in shape or size of areas designated as scar. This relative independence of amplitude on activation sequence in the abnormal regions may be due to the nature of propagation through low voltage areas. Conduction may take an irregular continuously changing path [14], so that any bipolar recording will sometimes be parallel and, at other times perpendicular regardless of the orientation of the recording bipole. In normal myocardium the direction of wavefront propagation might have greater effect because of the more homogeneously activated myocardium.

After identification of the infarct region, distinguishing portions of the infarct that contain surviving myocyte bundles from regions of dense, fibrous, inexcitable tissue that cause conduction block is desirable. A previous study proposed a pacing threshold of $>10 \mathrm{~mA}$ at a pulsewidth of $2 \mathrm{~ms}$ for this purpose and designated such sites as electrically unexcitable scar [5]. That previous study assessed electrogram amplitude during one activation sequence only and the findings are in agreement with our present study, where we found that although unexcitable scar was in low amplitude regions, it could not be identified based on electrogram amplitude alone with either activation sequence. In our present study we found that 
the vast majority of sites with low voltage were still excitable and on the other hand, very few sites with normal voltage were not excitable despite adequate catheter contact. This phenomenon could occur when the recording bipole was positioned at an inhomogeneous area where the distal bipole was at inexcitable scar whereas the proximal bipole was over surviving myocytes.

\section{Limitations}

Our approach was limited to one mapping catheter. Use of other interelectrode spacings and tip electrode size is likely to influence electrogram amplitude and may alter the impact of changing activation sequence on electrogram amplitude [15]. High pass filtering also reduces electrogram amplitude. Thus, the precise electrogram amplitudes associated with the infarct region in our study cannot be extrapolated to the use of catheters with different size electrodes or interelectrode spacing and filtering and needs further investigation in future studies. There are several caveats to the use of pacing for assessing underlying tissue excitability, including assessement of electrode stability and stimulus strength, which have been discussed previously [5,12,16-18].

\section{Conclusion}

Peak to peak bipolar electrogram amplitude used to create "voltage maps" of the ventricle is relatively insensitive to the direction of wavefront propagation when $\leq 1.5 \mathrm{mV}$ is considered the indication of abnormal areas, as previously established and now commonly used. Thus, voltage maps created with bipolar recordings in this manner are expected to be relatively robust depictions of abnormal ventricular regions despite variable catheter orientation and activation sequences that might be produced by different rhythms.

\section{References}

1. Callans DJ, Ren JF, Michele J, Girouard SD, Morris MM, Ideker RE. Electroanatomic left ventricular mapping in the porcine model of healed anterior myocardial infarction. Correlation with intracardiac echocardiography and pathological analysis. Circulation 1999;100:1744-1750.

2. Marchlinski FE, Callans DJ, Gottlieb CD, Zado E. Linear ablation lesions for control of unmappable ventricular tachycardia in patients with ischemic and nonischemic cardiomyopathy. Circulation 2000;101:1288-1296.

3. Ben Haim SA, Osadchy D, Schuster I, Gepstein L, Hayam $\mathrm{G}$, Josephson ME. Nonfluoroscopic, in vivo navigation and mapping technology Nat Med. 1996;2:1393-1395.

4. Shpun S, Gepstein L, Hayam G, Ben-Haim SA. Guidance of radiofrequency endocardial ablation with real-time three-dimensional magnetic navigation system. Circulation 1997;96:2016-2021.

5. Soejima K, Stevenson WG, Maisel WH, Sapp JL, Epstein LM. Electrically unexcitable scar mapping based on pacing threshold for identification of the reentry circuit isthmus: Feasibility for guiding ventricular tachycardia ablation. Circulation 2002;106:1678-1683.

6. Zeger SL, Liang KY. Longitudinal data analysis for discrete and continuous outcomes. Biometrics 1986;42:121130.

7. de Bakker JM, van Capelle FJ, Janse MJ, van Hemel NM, Hauer RN, Defauw JJ, Vermeulen FE, Bakker de Wekker PF. Macroreentry in the infarcted human heart: The mechanism of ventricular tachycardias with a "focal" activation pattern. J Am Coll Cardiol 1991;18:1005-1014.

8. Pogwizd SM, Hoyt RH, Saffitz JE, Corr PB, Cox JL, Cain ME. Reentrant and focal mechanisms underlying ventricular tachycardia in the human heart. Circulation 1992;86:1872-1887.

9. Gepstein L, Goldin A, Lessick J, Hayam G, Shpun S, Schwartz Y, Hakim G, Shofty R, Turgeman A, Kirshenbaum D, Ben-Haim SA. Electromechanical characterization of chronic myocardial infarction in the canine coronary occlusion model. Circulation 1998;98:2055-2064.

10. de Bakker JM, van Capelle FJ, Janse MJ, Wilde AA, Coronel R, Becker AE, Dingemans KP, van Hemel NM, Hauer RN. Reentry as a cause of ventricular tachycardia in patients with chronic ischemic heart disease: Electrophysiologic and anatomic correlation. Circulation 1988;77:589606.

11. Kornowski R, Hong MK, Gepstein L, Goldstein S, Ellahham S, Ben-Haim SA, Leon MB. Preliminary animal and clinical experiences using an electromechanical endocardial mapping procedure to distinguish infarcted from healthy myocardium. Circulation 1998;98:1116-1124.

12. Stevenson WG, Wiener I, Weiss JN. Comparison of bipolar and unipolar programmed electrical stimulation for the initiation of ventricular arrhythmias: Significance of anodal excitation during bipolar stimulation. Circulation 1986;73:693-700.

13. de Bakker J. Pathophysiological correlates of ventricular tachycardia in hearts with a healed infarct. In Zipes ed. Cardiac Electrophysiology: From Cell to Bedside. Saunders, 2000;415-422. 2004.

14. de Bakker JM, van Capelle FJ, Janse MJ, Tasseron S, Vermeulen JT, de Jonge N, Lahpor JR. Slow conduction in the infarcted human heart. 'Zigzag' course of activation. Circulation 1993;88:915-926.

15. Klitzner TS, Stevenson WG. Effects of filtering on right ventricular electrograms recorded from endocardial catheters in humans. Pacing Clin Electrophysiol 1990;13:69-77.

16. Meesmann M, Marchlinski FE. Effect of pacing current strength on indexes of myocardial activation in humans: Influence of chronic infarction and polarity of pacing. $J$ Am Coll Cardiol 1990;15:393-401.

17. Stevenson WG, Wiener I, Weiss JN. Contribution of the anode to ventricular excitation during bipolar programmed electrical stimulation. Am J Cardiol 1986;57:582-586.

18. Wikswo JP, Jr., Wisialowski TA, Altemeier WA, Balser JR, Kopelman HA, Roden DM. Virtual cathode effects during stimulation of cardiac muscle. Two-dimensional in vivo experiments. Circ Res 1991;68:513-530. 